\title{
New Permanent Magnet Synchronous Motor Current Sensing Phase Delay Compensation Method
}

\author{
Sei-Hun Park* and Il-Hwan Kim ${ }^{\dagger}$
}

\begin{abstract}
This paper presents a method that can improve the performance of permanent magnet synchronous motor current control by minimizing the measured current phase delay caused by the Low Pass Filter(LPF) used to cut off the noises that flowed in when feedback currents are measured. Although existing methods that change the Cutoff Frequency of the LPF can minimize phase delays during high speed rotations, their noise cutoff effects are much lower and this may lead to the decline of control performance. Therefore, in this study, an algorithm that can compensate current phase delays through relatively simple calculations from the synchronous motor $\mathrm{d}-\mathrm{q}$ axis coordinate transformation matrix and the inverse transformation matrix is proposed and the validity of the proposed method is verified by comparing the waveform of the calculated current with the waveform of actual currents through simulations and experiments.
\end{abstract}

Keywords: PMSM, Current sensing, Motor, VCF, Phase delay

\section{Introduction}

Although controlling a permanent magnet synchronous motors (PMSM) is more complicated compared to direct current (DC) motors, PMSMs have no problem in durability because they have no mechanical or electrical noise, maintain high efficiency over wide operating areas, and enable easy acceleration/speed change control such as speed control. [1-5] In the case of PMSM control systems actually used in industrial applications thanks to such characteristics, high speed acceleration/deceleration control and reduced vibrations or noises are required so that users' efficiency can be maximized. To this end, studies on the improvement of their performance in speed and position control and current control have been actively conducted. [6-16] Because speed controllers are generally designed assuming that current controllers operate ideally, to design accurate speed controllers, improving the performance of current controllers is very important.[11] In addition, to improve the performance of current controllers, accurate current measurement is a prerequisite. [12]

Meanwhile, low pass filters are used in many cases to cut off the noises that flow in during feedback current sensing for current control and this lead to the phenomenon of distortion of measured currents that is intensified as the cutoff frequency becomes lower. In particular, because of the characteristics of low pass filters, the phase of three phase current waveforms is delayed during high speed operation. This deteriorates the overall performance of

$\dagger$ Corresponding Author: Dept. of Electronic and Communication Engineering, Kangwon National University, Korea.

(ihkim@kangwon.ac.kr)

* Dept. of Electronic and Communication Engineering, Kangwon

National University, Korea. (seihun@kangwon.ac.kr)

Received: July 7, 2015; Accepted: October 15, 2014 current control and acts as a cause of system vibration and noises because this makes it impossible to make current commands synchronized with the position of the actual rotor. Therefore, techniques to prevent phase delays while cutting off the noises flowed in when currents are measured should be studied. [3]

In this paper, as a method of compensating the current phase delays caused by the low pass filter used to reduce noises during feedback current sensing for permanent magnet synchronous motor current control, a new current sensing delay compensation method for estimating actual phase currents without any phase delay using the current position and rotation speed of the rotor is proposed.

\section{Feedback Current Sensing Phase Delay Compensation Method}

For highly precise position and speed control for permanent magnet synchronous motors, a method that can reduce phase delays at high speed operation areas by adjusting the cutoff frequency of the LPF to prevent noises during current controller's feedback current sensing and solve the problem of phase delays has been studied. And the algorithm proposed in the present paper that can compensate current phase delays by estimating actual currents using the rotation speed of the motor and the position of the rotor has been studied.

\subsection{Variable Cutoff Frequency (VCF) method}

Control performance can be effectively improved in low speed operating areas through a method that cuts off noises by applying an LPF when currents are measured. 


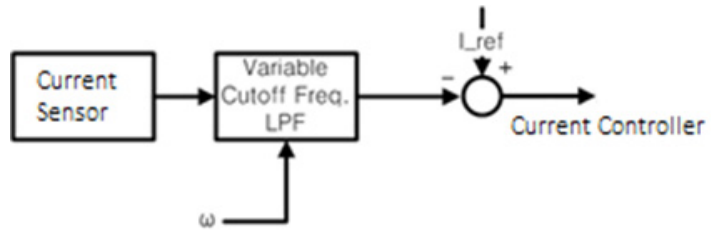

Fig. 1. Variable Cutoff Frequency structure

However, this method may rather deteriorate current control performance in high speed operating areas due to the phase delays caused by the LPF. One of methods for solving this problem is adjusting the cutoff frequency of LPF according to motor operation speed as with the configuration shown in Fig. 1.

This method changes the coefficient of the LPF according to the speed changes measured using the encoder attached to the motor to reduce noises in low speed areas to enable the implementation of effective control performance and minimize filtering in high speed areas to minimize feedback current phase delays. The Infinite Impulse Response (IIR) filter applied with Variable Cutoff Frequencies is expressed as shown by Eq. (1).

$$
y[n]=a x[n]+b y[n-1]
$$

In Eq. (1), $x[n]$ is the present input value of the measured current, $y[n]$ is the present output value after passing the LPF, $y[n-1]$ is the previous output value of $y[n]$, and $a$ and $b$ are the input coefficient and the feedback coefficient respectively. Current sensing phase delays can be compensated using Variable Cutoff Frequencies by setting the input coefficient and the feedback coefficient so that the sum of these two coefficients becomes 1 and changing the ratios of the two coefficients according to speed changes.

\subsection{Proposed current sensing phase delay compen- sation method}

In three phase synchronous motors, the relationship between the $d-q$ axis current expressed as a d-q synchronous coordinate system rotating at the speed of the rotor and the three phase current is as follows.

$$
\begin{aligned}
& I_{d q}^{e}=T(\theta) I_{a b c} \Rightarrow I_{a b c}=T(\theta)^{-1} I_{d q}^{e} \\
& T(\theta)=\frac{2}{3}\left[\begin{array}{ccc}
\cos \theta & \cos \left(\theta-\frac{2}{3} \pi\right) & \cos \left(\theta+\frac{2}{3} \pi\right) \\
-\sin \theta & -\sin \left(\theta-\frac{2}{3} \pi\right) & -\sin \left(\theta+\frac{2}{3} \pi\right)
\end{array}\right]
\end{aligned}
$$

From Eqs. (2) and (3), the relationships between the feedback currents of individual phases that passed the LPF and the actual currents after compensation of phase delays that is to be estimated can be calculated as follows.

$$
\begin{aligned}
& i_{a s}^{*}=I_{d s}^{e} \cos \theta-I_{q s}^{e} \sin \theta \\
& i_{a s}=I_{d s}^{e} \cos (\theta+\alpha)-I_{q s}^{e} \sin (\theta+\alpha) \\
& i_{b s}^{*}=I_{d s}^{e} \cos \left(\theta-\frac{2}{3} \pi\right)-I_{q s}^{e} \sin \left(\theta-\frac{2}{3} \pi\right) \\
& i_{b s}=I_{d s}^{e} \cos \left(\theta-\frac{2}{3} \pi+\alpha\right)-I_{q s}^{e} \sin \left(\theta-\frac{2}{3} \pi+\alpha\right) \\
& i_{c s}^{*}=I_{d s}^{e} \cos \left(\theta+\frac{2}{3} \pi\right)-I_{q s}^{e} \sin \left(\theta+\frac{2}{3} \pi\right) \\
& i_{c s}=I_{d s}^{e} \cos \left(\theta+\frac{2}{3} \pi+\alpha\right)-I_{q s}^{e} \sin \left(\theta+\frac{2}{3} \pi+\alpha\right)
\end{aligned}
$$

Where, $I_{a s}^{*}, I_{b s}^{*}, I_{c s}^{*}$ represent the currents of individual phases measured through the LPF, $I_{a s}^{*}, I_{b s}^{*}, I_{c s}^{*}$ represent the actual currents of individual phases that are to be estimated after compensation of phase delays, and represents the phase delays caused by the LPF.

Using Eq. (4), the estimated currents after compensation of actual phase delays can be calculated as follows.

$$
\begin{aligned}
& i_{a s}=i_{a s}^{*} \cos \alpha-\sin \alpha\left(I_{d s}^{e} \sin \theta+I_{q s}^{e} \cos \theta\right) \\
& i_{b s}=i_{b s}^{*} \cos \alpha-\sin \alpha\left(I_{d s}^{e} \sin \left(\theta-\frac{2}{3} \pi\right)+I_{q s}^{e} \cos \left(\theta-\frac{2}{3} \pi\right)\right) \\
& i_{c s}=i_{c s}^{*} \cos \alpha-\sin \alpha\left(I_{d s}^{e} \sin \left(\theta+\frac{2}{3} \pi\right)+I_{q s}^{e} \cos \left(\theta+\frac{2}{3} \pi\right)\right)
\end{aligned}
$$

Meanwhile, since the d-axis in the d-q synchronous coordinate system of permanent magnet synchronous motors is an axis where magnetic fluxes exist, the d-axis current is controlled to be 0. Therefore, Eq. (5) above is simplified as follows.

$$
\begin{aligned}
& i_{a s}=i_{a s}^{*} \cos \alpha-I_{q s}^{e} \cos \theta \sin \alpha \\
& i_{b s}=i_{b s}^{*} \cos \alpha-I_{q s}^{e} \cos \left(\theta-\frac{2}{3} \pi\right) \sin \alpha \\
& i_{c s}=i_{c s}^{*} \cos \alpha-I_{q s}^{e} \cos \left(\theta+\frac{2}{3} \pi\right) \sin \alpha
\end{aligned}
$$

On reviewing Eq. (6), it can be seen that the ratios of the measured current component $I_{a b c}^{*}$ and the current compensation component $I_{q s}^{e} \cos \theta$ are adjusted by that is changed according to rotation speeds. Meanwhile, since phase delays according to speeds can be obtained from the transfer function of the LPF, the actual currents can be estimated by applying the phase delays to the above equation.

\section{Simulation Results}

To check the effect to cut off noise inflows in the process of measurement of currents, the Variable Cutoff Frequency 


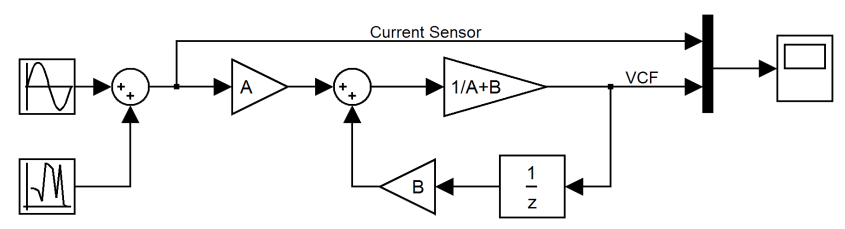

Fig. 2. Block diagram of simulations of the VCF method
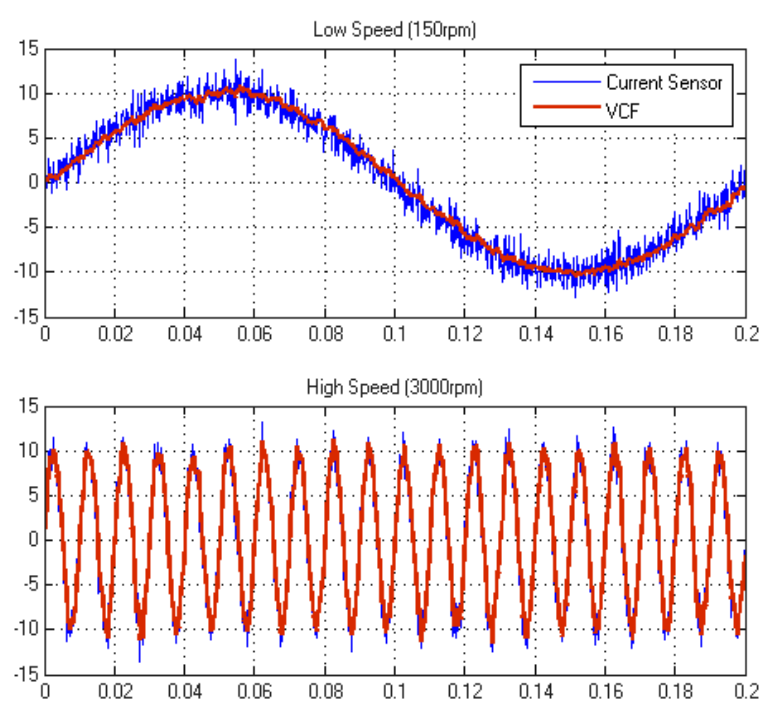

Fig. 3. Simulated current waveforms of the VCF method according to speeds

method and the phase delay compensation algorithm proposed in this paper are checked through simulations using MATLAB/ Simulink.

\subsection{Variable cutoff frequency method}

First, a block diagram of the MATLAB/Simulink for simulations of the Variable Cutoff Frequency method that prevents phase delays by adjusting the coefficient of the filter according to speeds is as shown in Fig. 2.

Currents consisting of sine waves added with random noises become to pass through the IIR filter shown in Eq. (1) that provides feedback and the cutoff frequency is changed be adjusting the values of Gain A which is the ratio of the present input and Gain B which is the ratio of the previous output. Changes in the output current waveform following the changes in the Cutoff Frequency of the LPF are as shown in Fig. 3. As the ratios of Gain A to Gain B which is the ratio of the previous output, 1:7 was applied in low speed areas and 5:5 was applied in high speed areas.

On reviewing the waveforms in Fig. 3, it can be seen that the noises in the measured current were effectively removed in the low speed area. In the high speed area, it can be seen that although noise inflows were not cut off because the Cutoff Frequency was changed, almost no phase delay occurred. As shown above, by applying Variable Cutoff Frequencies, the problem of noise inflows

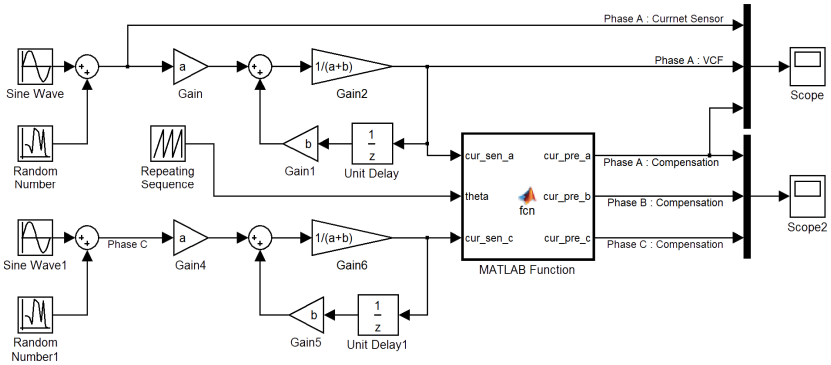

Fig. 4. A block diagram of a simulation of the proposed current sensing phase delay compensation

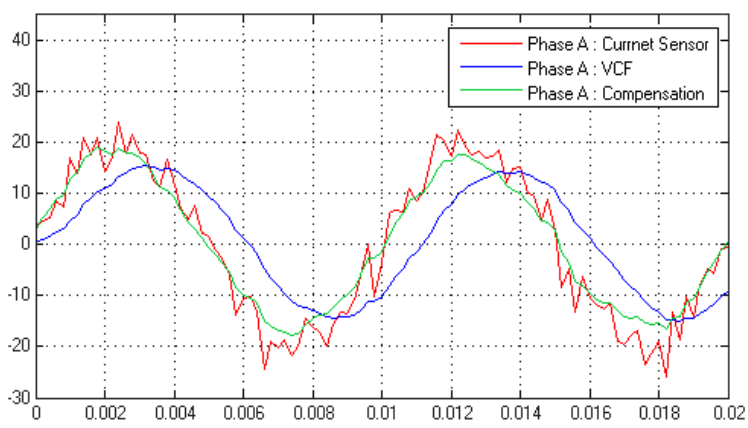

Fig. 5. Simulated waveform of a phase currents in high speed areas

in low speed areas can be solved and the problem of phase delays in high speed areas can be also solved to some extent.

\subsection{The proposed current sensing phase delay com- pensation method}

A block diagram of the MATLAB / Simulink for checking the operation of the phase delay compensation algorithm proposed in this paper which is an improved current measuring method using Eq. (6) is as shown in Fig. 4.

Since the phase delays caused by the LPF are calculated using the motor's rotation speed and the angle of the rotor, actual phase currents are estimated and compensated. The results of the simulations of current waveforms in high speed operating areas are shown in Fig. 5.

In Fig. 5, it can be seen that, although phase delays are not sufficiently compensated in the phase compensation method using the VCF, the phase delays caused by the LPF were effectively compensated in the phase delay compensation algorithm proposed in this paper so that actual current waveforms were estimated closely.

\section{Experiment}

\subsection{Configuration of the experimental system}

An experimental system was configured as shown in 


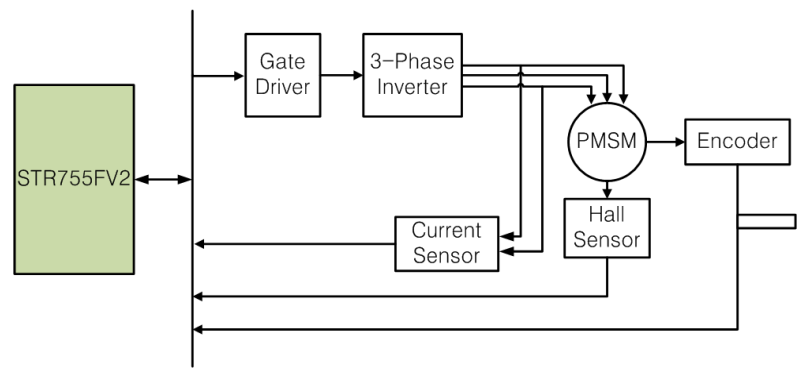

Fig. 6. Block diagram of the experimental system

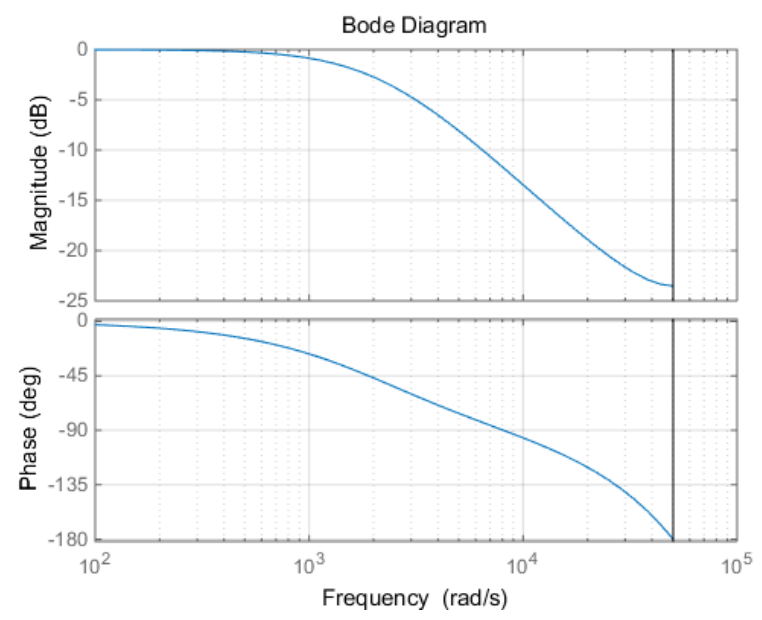

Fig. 7. Bode diagram of the designed IIR filter

Fig. 6 to check the usefulness of the current estimation algorithm proposed in this paper and whether current control performance was improved.

The experimental system was implemented using an STR755FV2 of STMicro Co. and the sampling frequency was set to $16 \mathrm{kHz}$. The motor used in the experiment was a 500W 4pole PMSM and a hole sensor and an encoder were also used for rotor position sensing. An industrial sewing machine was used as a load and the maximum rotation speed in loaded states is approximately 3800RPM.

\subsection{Variable Cutoff Frequency method}

To apply the LPF Variable Cutoff Frequency method, a primary digital IIR filter was designed as shown by Eq. (7). Configuration of the experimental system

$$
\begin{gathered}
y[n]=\frac{1}{8} x[n]+\frac{7}{8} y[n-1] \\
H(z)=\frac{0.125}{z-0.875}, \text { sample time }: 0.0000625 \mathrm{~s}
\end{gathered}
$$

Before applying the experimental system, the characteristics of the filter were checked using MATLAB in order to find out the sizes of the phase delayed when the above filter was applied according to motor rotation speeds and the resultant filter characteristics are as shown in Fig. 7.

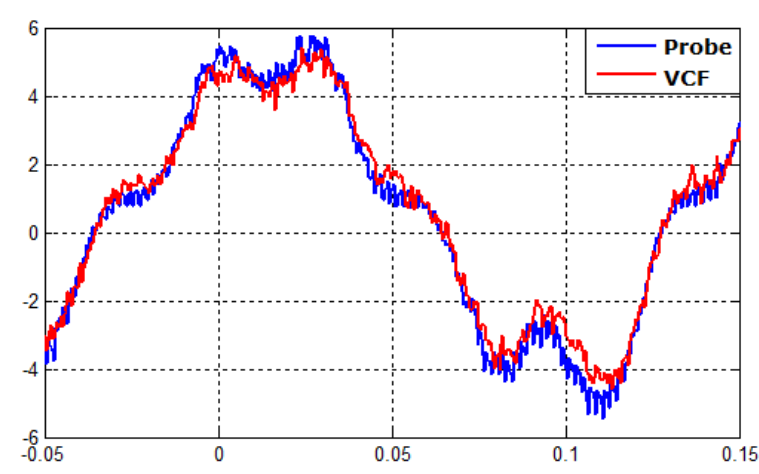

Fig. 8. Waveforms of currents applied with the VCF method in low speed areas

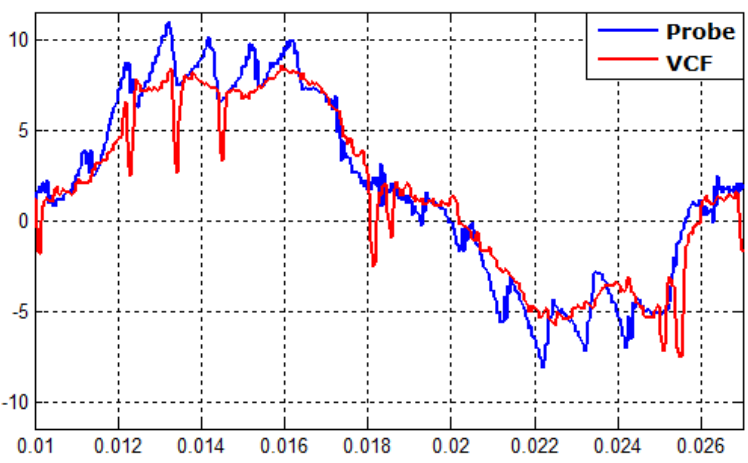

Fig. 9. Waveforms of currents applied with the VCF method in high speed areas

On reviewing changes in phase delay according to rotor speeds in the Bode diagram, it can be seen that phase delays of approximately $30^{\circ}$ occur at $3800 \mathrm{RPM}$ which is the maximum speed of the experimental system.

The waveform of the currents measured in real time in low speed areas and high speed areas while changing the Cutoff Frequencies obtained through simulations using software according to the motor speeds obtained through the Incremental Encoder and the waveform of the currents flowing through actual phases measured using current probes were compared with each other in low speed areas and high speed areas as shown in Figs. 8 and 9.

As shown in Fig. 8, it can be seen that noise inflows were effectively cut off in low speed areas by maximizing the filtering by the LPF. Through the cutoff, attenuation of the motor noises and vibrations occurring due to the noises that flowed in low speed areas can be expected. However, as shown in Fig. 9, it can be seen that, in high speed operating areas, although phase delays were reduced, the cutoff is not very effective on noise inflows. When this Variable Cutoff Frequency method is used, although the noises that flowed in during high speed operation are not much problematic in the case of applications with large mechanical loads because such systems have very large inertial energy when operated at high speeds, such noises can cause problems to systems that have very small 


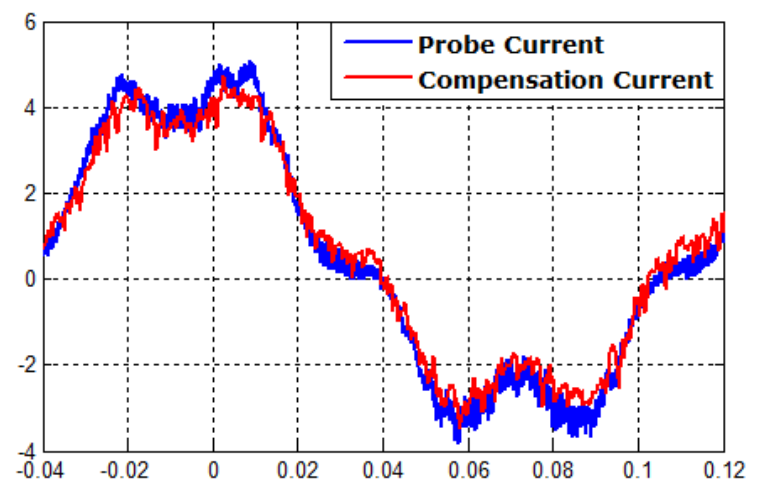

Fig. 10. Comparison between the waveform of the probe current and that of the phase delay compensation current (low speed)

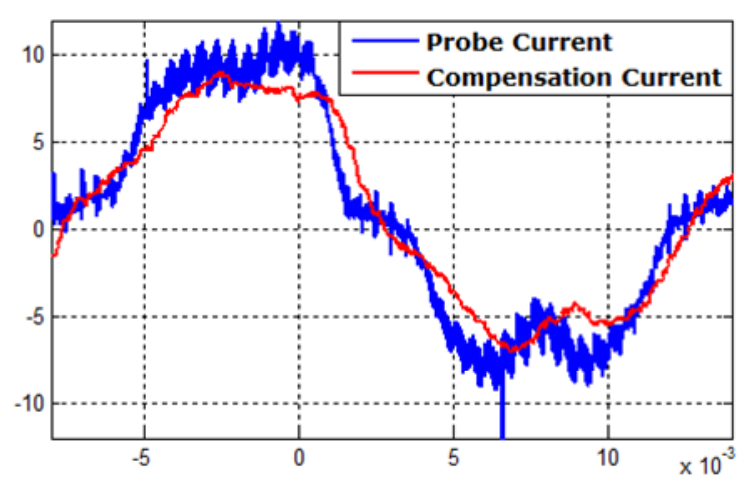

Fig. 11. Comparison between the waveform of the probe current and that of the phase delay compensation current (medium speed)

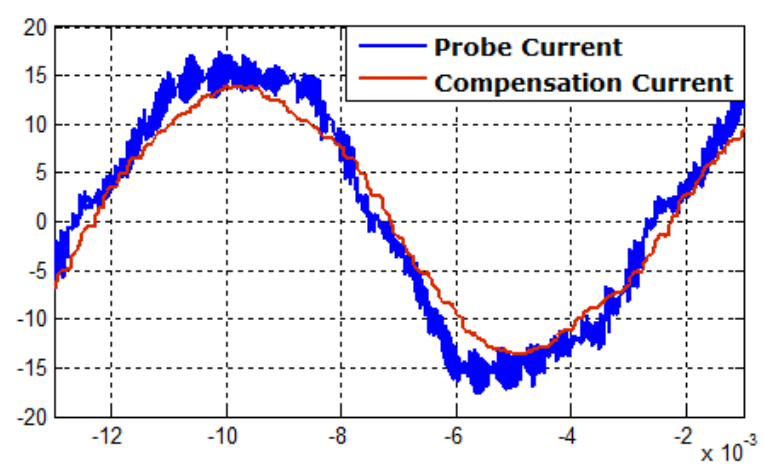

Fig. 12. Comparison between the waveform of the probe current and that of the phase delay compensation current (high speed)

mechanical loads and require high speed rotation.

\subsection{The proposed current sensing phase delay compensation method}

The results of experiments conducted by applying the phase delay compensation algorithm proposed in this paper are shown in Figs. 10, 11, and 12.
From the results of experiments of the phase delay compensation algorithm proposed in this paper, it could be seen that the currents flowing through actual coils could be estimated with almost no phase delay regardless of motor rotation speeds. In addition, the proposed method also showed effects to cut off the noises in high speed areas that were problematic in the method using the VCF.

\section{Conclusion}

In this paper, a method was presented that can improve the performance of permanent magnet synchronous motor current control by minimizing the phase delays in measured currents caused by the LPF used to cut off the noises that flow in when feedback currents are measured. Although the existing Variable Cutoff Frequency method minimized phase delays by finding Cutoff Frequencies suitable for changed speed areas through simulations and comparing the waveform of the estimated currents with that of actual currents through experiments, the method was shown to have much lower noise cutting effects in high speed areas.

Therefore, in this study, an algorithm that can compensate current phase delays through relatively simple calculations from the synchronous motor $\mathrm{d}-\mathrm{q}$ axis coordinate transformation matrix and the inverse transformation matrix was proposed and the validity of the proposed method was verified by comparing the waveform of the calculated current with the waveform of actual currents through simulations and experiments. It was identified that the currents estimated through this algorithm followed the currents of actual coils with similar waveforms and that the noise cutting effects that were problematic in the VCF method well appeared in the proposed method regardless of changes in speeds.

When currents are measured, the algorithm proposed in this paper can be applied to minimize differences between actual phase currents and measured currents to improve the performance of permanent magnet synchronous motor current control thereby improving vibrations and noises in diverse applications.

\section{Acknowledgements}

This study was supported by 2013 Research Grant from Kangwon National University (No. 120131282)

\section{References}

[1] M. H. Shin, D. S. Hyen, S. B. Cho and S. Y. Choe, "An improved stator flux estimation for speed sensorless stator flux orientation control of induction 
motors", IEEE PESC, pp. 1581-1586, 1998.

[2] K. Y. Nam, W. T. Lee, C. Lee, and J-Pyo Hong, "Reducing torque of DC motor by varying input voltage”, IEEE Trans. Mag. vol. 42, pp. 1307-1311, 2006.

[3] L. Zhang and W. L. Qu, "Commutation torque ripple restraint in BLDC motor over whole speed range", in Proc. IEEE ICEMS, pp. 1501-1507. 2005.

[4] Y. Liu, Z. Q. Zhu, and D. Howe, "Direct torque control of brushless DC drives with reduced torque ripple," IEEE Trans. Ind. Appl., vol. 41, no. 2, pp. 599-608, 2005.

[5] Y. Liu, Z. Q. Zhu, and D. Howe, "Instantaneous torque estimation in sensorless direct torque controlled brushless DC motors," IEEE Trans. Ind. Appl., vol. 42, no. 5, pp. 1275-1283, 2006.

[6] Z. Q. Zhu, Y. Liu, and D. Howe, "Steady-state dynamic performance of a direct torque controlled PM brushless DC drive accounting for influence of PWM chopping and cogging torque", in Proc. IEE Int. Conf. Power Electron., Mach. and Drives, Dublin, pp. 556-560, 2006.

[7] Tae-Sung Kim, Sung-Chan Ahn, and Dong-Seok Hyun, "A new current control algorithm for torque ripple reduction of BLDC motors," IECON '01, The 27th Annual Conf. of the IEEE, vol. 2, pp. 1521-1526, 2001.

[8] Joong-Ho Song and Ick Choy, "Commutation torque ripple reduction in brushless DC motor drives using a single DC current sensor", IEEE Trans. of Power Electronics, vol. 19, pp. 312-319, 2004.

[9] K. Chung, Y. Zhu, I. Lee, K. Lee and Y. Cho, "Simulation of the Reduction of Force Ripples of the Permanent Magnet Linear Synchronous Motor," Journal of E.E.T. vol. 2, pp. 208-215, 2007.

[10] J. Holtz and L. Springob, "Identification and compensation of torque ripple in high-precision permanent magnet motor drives," IEEE Trans. Ind. Electron., vol. 43, no. 2, pp. 309-320, 1996.

[11] T. M. Jahns and W. L. Soong, "Pulsating torque minimization techniques for permanent magnet AC motor drives - a review," IEEE Trans. Ind. Electron., vol. 43, no. 2, pp. 321-330, 1996.

[12] B. Kang, C. Kim, H. Mok and G. Cheo, "Analysis of Torque Ripple in BLDC Motor with Commutation Time," Proceedings of ISIE 2001, pp. 1044-1048, 2001.

[13] T. Kim, S. Ahn and D. Hyun, "A New Current Control Algorithm for Torque Ripple Reduction of BLDC Motors," Proceedings of IECON '01, pp. 1521-1526, 2001.

[14] K. Lee, J. Park, H. Yeo and J. Yoo, "Current Control Algorithm to Reduce Torque Ripple in Brushless DC Motors," Proceedings of ICPE' 98, pp. 380-385, 1998.

[15] Y. Liu, Z. Q. Zhu and D. Howe, "CommutationTorque-Ripple Minimization in Direct-Torque-Con- trolled PM Brushless DC Drives," IEEE Trans. Ind. Applicat., vol. 43, no. 4, pp. 1012-1021, 2007.

[16] H. Lu, L. Zhang and W. Qu, "A New Torque Control Method for Torque Ripple Minimization of BLDC Motors With Un-Ideal Back EMF," IEEE Trans. Power Electronics, vol. 23, no. 2, pp. 950-958, 2008.

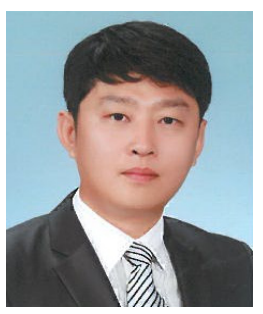

Sei-Hun Park He received B.S. and M.S. degree in degree in the Department of Electrical and Telecommunication Engineering from Kangwon National University, Korea, in 2007 and 2009, respectively. He is currently a Ph.D candidate in the Department of Electrical and Telecommunication Engineering from Kangwon National University. His research interests are automotive control, robust control and power, filtering and signal processing.

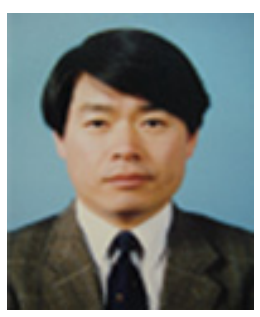

Il-Hwan Kim He received B.S. and M.S. degree in Control and Instrument Engineering from Seoul National University in 1982 and 1985 respectively and Ph.D. at the Tohoku University in 1993. In 1995, he joined the Department of Electrical and Electronic Engineering at the Kangwon National University and is currently a professor. His research interests include control, mechatronics, and human interfaces. 